\title{
Correction to: Smart Cyclones: Creating Artificial Cyclones with Specific Intensity in the Dearth Situations Using IoT
}

\author{
G. Subbarao, S. Hrushikesava Raju, Lakshmi Ramani Burra, \\ Venkata Naresh Mandhala, and P. Seetha Rama Krishna
}

Correction to:

Chapter "Smart Cyclones: Creating Artificial Cyclones with Specific Intensity in the Dearth Situations Using IoT" in: S. K. Saha et al. (eds.),

Smart Technologies in Data Science and Communication, Lecture Notes in Networks and Systems 210, https://doi.org/10.1007/978-981-16-1773-7_28

In the original version of the chapter, the following belated corrections have been incorporated:

The author name "S. Hrushikesava Rao" has been changed to "S. Hrushikesava Raju" in the Frontmatter, Backmatter and in Chapter "Smart Cyclones: Creating Artificial Cyclones with Specific Intensity in the Dearth Situations Using IoT”.

The correction/erratum chapter and the book have been updated with the change. 